\title{
Design Analysis and Human Tests of Foil based Wheezing Monitoring System for Asthma Detection
}

\author{
Sherjeel M. Khan ${ }^{1}$, Nadeem Qaiser ${ }^{1}$, Sohail F. Shaikh ${ }^{1,}$ and Muhammad Mustafa Hussain, Senior Member, IEEE ${ }^{1,2 *}$ \\ ${ }^{1}$ mmh Labs, CEMSE, King Abdullah University of Science and Technology (KAUST), Thuwal, Saudi Arabia \\ ${ }^{2}$ EECS, University of California, Berkeley, USA \\ muhammadmustafa.hussain@kaust.edu.sa1',mmhussain@,berkeley.edu²
}

\begin{abstract}
We present a flexible acoustic sensor that has been designed to detect wheezing (a common symptom of asthma) while attached to the chest of a human. We adopted a parallel plate capacitive structure using air as the dielectric material. The pressure (acoustic) waves from wheezing vibrate the top diaphragm of the structure, thereby, changing the output capacitance. The sensor is designed such that it resonates in the frequency range of wheezing $(100-1000 \mathrm{~Hz})$ which presents twofold benefits. The resonance results in large deflection of the diaphragm that eradicates the need for using signal amplifiers (used in microphones). Secondly, the design itself acts as a low pass filter to reduce the effect of background noise which mostly lies in $>1000 \mathrm{~Hz}$ frequency range. The resulting analog interface is minimal and thus, consumes less power and occupies less space. The sensor is made up of low-cost sustainable materials (aluminum foil) which greatly reduces the cost and complexity of manufacturing processes. A robust wheezing detection (Matched filter) algorithm is used to identify different types of wheezing sounds among the noisy signals originating from the chest that lie in the same frequency range as wheezing. The sensor is connected to a smartphone via Bluetooth, enabling signal processing and further integration into digital medical electronic systems based on the Internet of Things (IoT). Bending, cyclic pressure, heat, and sweat tests are performed on the sensor to evaluate its performance in simulated real-life harsh conditions.
\end{abstract}

Keywords - wheezing; wireless; foil; low-cost; healthcare; flexible.

\section{INTRODUCTION}

About 334 million people were affected by asthma as of 2014 , and the number continues to climb [1,2]. If a patient experiences three or more wheezing episodes in a year, they are diagnosed with active asthma [3]. A study showed that asthma can be diagnosed at an early stage using non-invasive practices by monitoring the airway resistance in the trachea [4]. This airway resistance produces wheezing characterized by musical, sinusoidal sounds superimposed on breathing with frequencies of $100-1000 \mathrm{~Hz}$ and a duration of $>250 \mathrm{~ms}$ [5-9]. Similar to the behavior of sound, wheezing travels through a medium in the form of pressure fluctuations. Thus, an acoustic (pressure) sensor can be used to detect wheezing.

A common method used in health monitoring wearables for wheeze detection is electrocardiography (ECG). [10]. The output voltage signal from the ECG electrodes is in the order of a few millivolts. Thus, they require amplification and signal condition circuitry before the data can be fed to a microprocessor. This increases the size and power consumption of the overall system [11]. Soft materials have been used to detect human vocalization using muscle movements. For example, woven graphene fabric are used to monitor throat muscle movement in response to sounds originating from the neck [12]. Some other flexible sensors used to collect reliable acoustic data include single-walled carbon nanotube (SWNCT) embedded in a hydrogel [13] and nanowires grown on polytetrafluoroethylene (PTFE) films [12]. However, additional sensors are required to interpret their data. Additionally, the graphene/CNT-based sensors require complex fabrication processes that increase the cost.

Microphones have proven to be the most practical solution to acquire sounds from the neck or chest [14-17]. They are placed on the neck to monitor vital signs and ingestion habits [17]. However, microphones based wearable are rigid, which makes them less comfortable to use for long intervals of time as a disease monitoring wearable [18]. The sensors have small dimensions to reduce the high costs associated with siliconprocessing equipment and the silicon material itself. It results in a high resonance frequency (in the $\mathrm{kHz}$ range) diaphragm with small output signals [19]. Thus, they require signal amplification circuits, which introduce additional noise in the system that must be reduced by added signal conditioning circuits $[20,21]$. The compromise of using a higher resonance frequency diaphragm is made so that the sensor can respond to the large audible frequency range of an average human $(20 \mathrm{~Hz}-$ $20 \mathrm{kHz}$ ). This is desirable for microphones as they are intended to record human speech. However, in the case of wheezing, it is more beneficial to have a specialized acoustic sensor that is low-cost, minimalistic, consumes less power, and responds in the wheezing frequency range $(100 \mathrm{~Hz}-1000 \mathrm{~Hz})$. For that, we need a large diaphragm made with low-cost materials to get a low resonance frequency with a high signal to noise ratio.

Here we present a low-cost, flexible wheezing sensor made from aluminum foil using low-cost and scalable manufacturing techniques. The sensor has a parallel plate capacitive sensor structure with an air gap acting as the dielectric. The sensor is designed such that the resonance frequency of the diaphragm lies in the desired wheezing frequency range. Consequently, the diaphragm of the sensor is able to achieve a sizable deflection, creating a large output signal without the use of amplification circuits. The sensor is directly connected to a microcontroller, which wirelessly transmits raw data to a peripheral device such as a smartphone/PC for signal processing and wheezing detection. We have previously shown that such a sensor can be used to detect wheezing sounds from the chest [1]. The flexibility and versatility of the sensor allow it to be packed in a flexible Styrofoam packaging to cancel out the effects of background noise. It adheres to the chest of a human subject with minimal intrusion or discomfort. The other chest sounds 


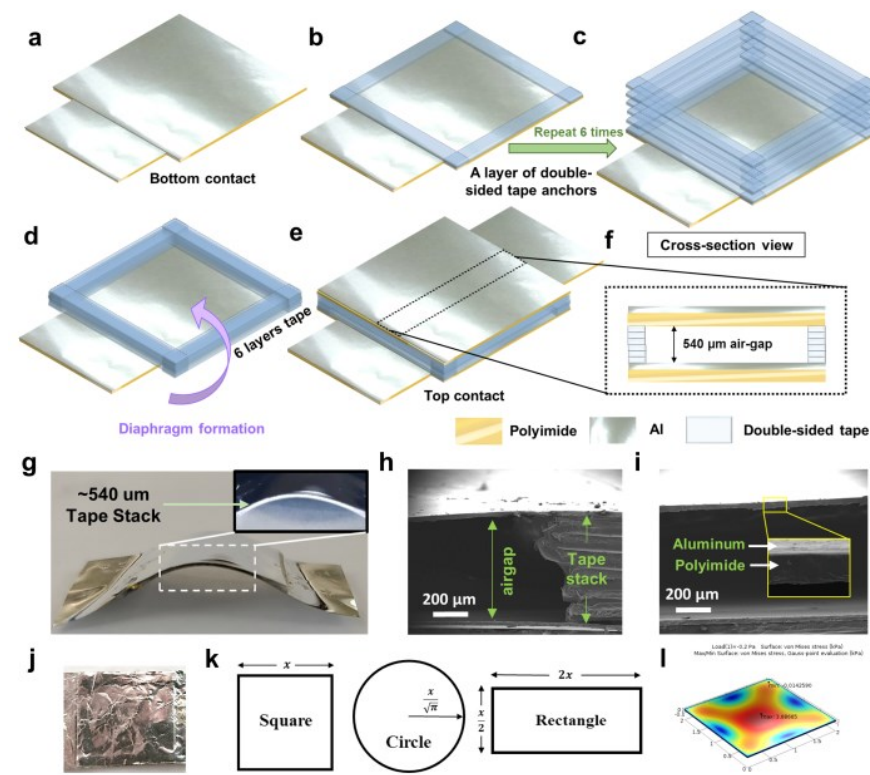

Fig. 1. Fabrication of the sensor. (a) Cutting the sheet to make bottom contact. (b) Placing a thin layer of $90 \mu \mathrm{m}$ double-sided tape at the edges. (c) Repeating the step six times to get a $540 \mu \mathrm{m}$ height. (d) Placing the top contact in line with the edges of the bottom plate and the double-sided tape. (e) Illustration of the fabricated sensor. (f) Cross-section view of the sensor showing how the tape creates an air space between the two sheets. (g) Actual fabricated sensor bent to show the flexibility of the sensor; the inset highlights the small gap between the two sheets in a negative and zoomed image. (h) SEM image of the sensor edge showing the tape stack and air gap. (i) SEM image of the sensor center with yellow box zooming in on top contact of the diaphragm. (j) Crumpling of $\mathrm{Al}$ foil-based sensor after normal handling. (k) Areas of square, circle, and rectangle diaphragms were equal to each other in terms of $x$. (1) FEM simulation result of stress analysis on the square diaphragm. (Modified from [1])

act as noise during wheezing detection like coughing, breathing, and talking. We recorded several chest sounds using our sensor and superimposed them on wheezing sounds to create noisy signals. A robust signal processing method is used to detect wheezing from the noisy signal. The sensor is attached to the chest of a human subject to investigate a scenario in which sounds are detected in real time from the human chest. The mechanical integrity of the flexible sensor is evaluated in bending, cyclic pressure, heat, and sweat exposure tests.

\section{DESIGN AND FABRICATION}

\section{A. Fabrication of Wheezing Sensor}

Sound traverses in the air in the form of pressure fluctuations produced by acoustic waves [22]. We have thus created an acoustic sensor in the form of a parallel plate capacitor where an air gap between the two parallel plates acts as the pressuresensitive dielectric material. Acoustic waves put pressure on the top movable diaphragm, thereby, reducing the thickness ' $d$ ' of the air gap which in turn increases the output capacitance ' $C$ ', as expressed by Equation 1 [23].

$$
C=\varepsilon_{0} \varepsilon_{r} A / d
$$

Where $\varepsilon_{\mathrm{r}}=1.0006$ and $\varepsilon_{0}=8.854 \times 10^{-12} \mathrm{~F} \cdot \mathrm{m}^{-1}$. Due to the high compressibility of air, commercial parallel plate based accelerometers also use air gap as the pressure sensitive dielectric material $[24,25]$. The high compressibility factor of air allows the air gap based capacitive structure to respond to the extremely low pressures of acoustic waves.

We have adopted a low-cost method, compatible with largescale manufacturability, to make the sensor structure. An illustration of the method is outlined in Fig. 1. The air gap is created by creating pillars of stacked double tape around the edges of a metal foil cut in a rectangular shape (Fig. 1a). A stack of six layers of double tape (where each strip is $1.2 \mathrm{~mm}$ wide and $\sim 90 \mu \mathrm{m}$ thick) creates a $\sim 540 \mu \mathrm{m}$ gap which is sufficient for the diaphragm to freely vibrate in the air gap (Figs. 1b-d). A second rectangular piece of metal foil is placed on top of the tape stack to complete the parallel plate capacitive structure (Figs. 1e-f). The thin flexible metal foil allows the formation of a flexible sensor that can conform to irregular shapes while retaining the air gap (Fig. 1g). Cross-sectional SEM (Scanning Electron Microscopy) images reveal that the height is actually $626 \mu \mathrm{m}$ at the edges due to variability in the thickness of double-sided tape adhesive (Fig. 1h). In the center, the weight of the diaphragm reduces the air gap to $510 \mu \mathrm{m}$ (Fig. 1h).

\section{B. Diaphragm Material, Size, and Shape Selection}

We aim to design our sensor such that it resonates within the frequency range of wheezing in order to achieve the maximum output signal. While the frequency of a wheeze from the trachea lies in the range of $100-2500 \mathrm{~Hz}$, it is reduced to $100-1000 \mathrm{~Hz}$ from the chest because lung tissue, chest wall, and skin absorb the higher frequencies before they reach our sensor $[5,6]$. The chest-wall tissue acts as a low-pass spectral constraint on respiratory sounds, which, when measured on the skin surface with an acoustic sensor (microphone or accelerometer) positioned on human chest, back or neck, typically reside in the frequency band below $1 \mathrm{kHz}$ [26]. Excess amount of chest hair may cause interference with the adhesion and performance of the sensor. The region of the neck close to the trachea could be a suitable alternative location to acquire wheezing sounds.

\section{1) Diaphragm Material}

Aluminum foil is cheap, readily available, and responds reliably to pressure. Although it is made up of metal, aluminum foil tends to crumble and deform very easily after undergoing normal wear and tear due to its small thickness. A thin metal film has less elastic strength and bends easily, as the elastic modulus is inversely proportional to the cross-sectional surface area [27]. The crumpling (plastic deformation) of the aluminum metal film after prolonged usage can be observed in Fig. 1j. Thus, we tested different diaphragm materials: copper $(\mathrm{Cu})$ foil, aluminum (Al) foil, Al on PET (Polyethylene terephthalate), and $\mathrm{Al}$ on polyimide $\mathrm{PI}$ ) to find a more versatile metal foil. The $\mathrm{Al}$ and $\mathrm{Cu}$ metal foils showed buckling and dents after prolonged usage. Al on PET and PI showed higher mechanical strength due to the mechanical rigidity provided by polymers. Al on PI exhibited maximum displacement with the application of pressure. Therefore, we decided to use the commercially available Al metalized PI film, Liren's LR-PI 100AM, in order to improve the strength of the diaphragm while maintaining the useful characteristics of aluminum foil. PI is a flexible substrate with high thermal and mechanical stability that acts as a supporting and carrier film for the $\mathrm{Al}$, while $\mathrm{Al}$ provides the relevant conductivity necessary for the formation of a capacitor. Also, Liren's LR-PI 100AM is thin, consisting of a $200 \mathrm{~nm}$ thick $\mathrm{Al}$ on top of a $25 \mu \mathrm{m}$ thick PI. Studies have shown that a thin diaphragm results in an increased deflection of the diaphragm while a thinner $\mathrm{Al}$ on a PI film increases the overall tensile strength $[28,29]$. In addition, the presence of $\mathrm{Al}$ on PI improves the linear elastic range of PI.

\section{2) Diaphragm Shape}




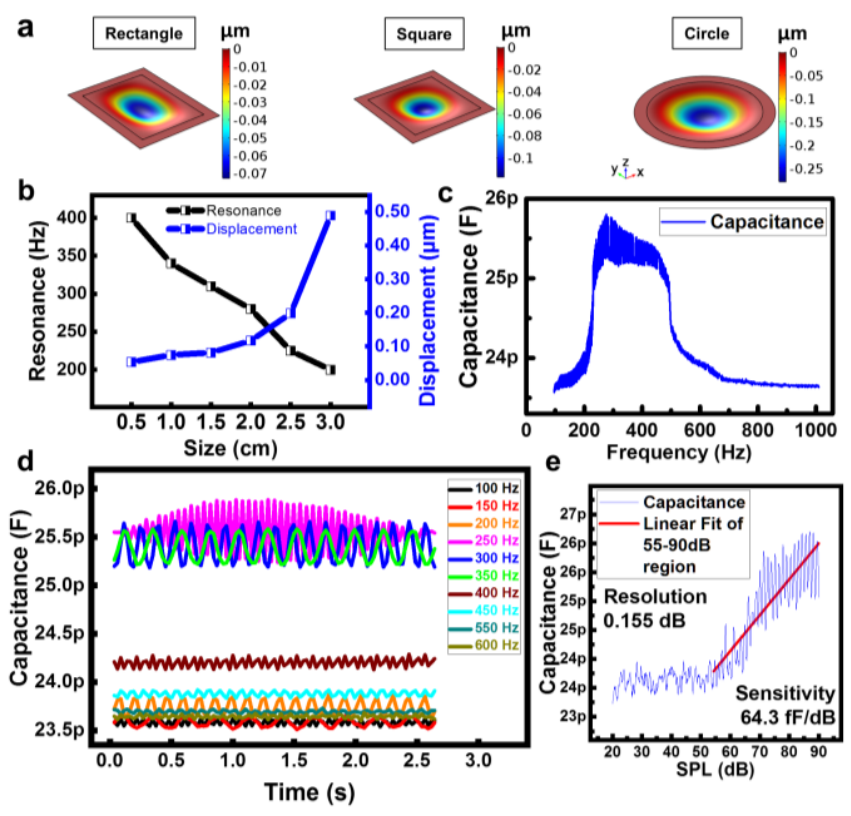

Fig. 2. Comparison of deflection among (a) rectangular, square, and circular diaphragms having the same area. (b) Variance in resonance frequency and maximum displacement of the diaphragm for various sizes of the square diaphragm. The x-axis shows the length of each side of the square-shaped diaphragm. (c) The output response of the sensor for a sweep of sound frequencies from 100 to $1000 \mathrm{~Hz}$. (d) The output of sensor at various frequencies with the acoustic resonance phenomenon observed at $250 \mathrm{~Hz}$. (e) Sensor response to increasing sound intensity to calculate sensitivity.

We created a model of the Al on PI metal foil in COMSOL. Simulations of different sized diaphragms showed that the shape affects the extent deflection for a given pressure. Shape analysis studies have previously been performed on the silicon diaphragms used in microphones [30,31]. We used the finite element method (FEM) in COMSOL to compare displacements of circle, square, and rectangular shaped diaphragms in order to validate that the findings for silicon also hold for the $\mathrm{Al}$ on PI foil. The surface area of the three different diaphragm shapes was kept constant defined in terms of a variable ' $x$ ', where ' $x$ ' is the side length of the square diaphragm. The dimensions of each shape in terms of ' $x$ ' can be seen in Fig. 1k. For the FEM simulations in COMSOL, we chose an ' $x$ ' of $1 \mathrm{~cm}$. We put all the diaphragm shapes under an equivalent sound pressure level (SPL) intensity, $80 \mathrm{~dB}$, which was the sound intensity of a forced cough measured from the chest of a 28 -year-old adult male, in a quiet environment of $28 \mathrm{~dB}$ background noise, using the Android application "Sound Meter". In order to find the equivalent value of pressure in pascals $(\mathrm{Pa})$, we used the formula shown in Equation 2 [22] :

$$
\text { Sound Pressure }(d B)=20 \log _{10} \frac{\text { Measured Sound Pressure }}{\text { Reference Pressure }}
$$

where the reference pressure was set to $20 \mu \mathrm{Pa}$, which is the threshold of human hearing [32]. An SPL of $80 \mathrm{~dB}$ corresponds to $0.2 \mathrm{~Pa}$. We calculated the deflection at the center of each diaphragm using a pressure of $0.2 \mathrm{~Pa}$ applied to the diaphragm (Fig. 2a). The circular diaphragm has the largest deflection, while the rectangular diaphragm showed the smallest deflection. The circular diaphragm may seem like the best choice to get a large deflection. However, keeping the material waste and manufacturing costs to a minimum is equally important in order to realize a low-cost sensor. We have included a supplementary color MPEG file (S1) which shows that the square-shaped diaphragms are much faster to produce than the circular diaphragms, and less material is wasted. This is available at http://bit.ly/2Ks4Weo. Thus, we chose the square shape for our sensors diaphragm which had the second-best deflection. According to the specifications, the tensile strength of Liren's LR-PI 100AM is $>140 \mathrm{MPa}$. The stress induced by an $80 \mathrm{~dB}$ sound (of cough), which we expected to be the upper limit of sound that would be experienced by the sensors attached to the chest, was also calculated using FEM simulations. The results of the simulation, as seen in Fig. 11, show that the point of maximum stress is at the center of the square diaphragm with surface stress of $3.88 \mathrm{kPa}$, which is well below the chosen diaphragm material's tensile strength.

\section{3) Diaphragm Size}

Diaphragms with a larger surface area have been mathematically proven to result in a larger deflection [33]. Larger diaphragms in commercial microphones are more sensitive and more responsive to sounds than small diaphragms, as seen in large-diaphragm vs. small-diaphragm condenser models (LDC or SDC, respectively) [19]. The changes in capacitances are larger in LDC models, thus providing a larger signal-to-noise ratio than SDC making LDC the better choice for sound recording. Furthermore, LDC has a smaller transient response to changes in sound pressure [34]. Studies show that the resonance frequency decreases with an increase in the size of the diaphragm [35], i.e., LDC microphones have a lower resonance frequency due to their higher diaphragm mass. The useful features of an LDC confirm that we need a large-sized diaphragm in order to get the best wheezing sensor. The thickness of the diaphragm also affects the deflection and resonance frequency [31]. LDC microphones, thus, suffer from a proximity effect as the sound intensity falls significantly with increasing distances [36]. Therefore, we need to keep the distance between the sensor and the sound source small. This sensor is hooked directly into our PSoC which has been smartly optimized to perform fast data acquisition while also being able to send the data over Bluetooth using the same chip.

Since we used a metal foil with a fixed thickness cut in a square shape, we can only optimize the size of the diaphragm to achieve the balance between the deflection and the resonance frequency. FEM simulations showed that the resonance frequency of the chosen metal foil diaphragm lies in sub $500 \mathrm{~Hz}$ range for sizes less than $3 \mathrm{~cm}$. The deflection was calculated from FEM simulations of the diaphragm, while the resonance frequency was found out by performing experiments. In order to find the optimal diaphragm size, we investigated how both deflection and frequency changed as the side lengths of the square diaphragm varied from 0.5 to $3.0 \mathrm{~cm}$ (Fig. 2b). Our results concur with similar studies that showed an increase in deflection and a decrease in resonance frequency as the diaphragm size is increased [35, 37, 38]. As evident from Fig. $\mathbf{2 b}$, the sensor diaphragms with side lengths of $1-3 \mathrm{~cm}$ had a resonance frequency within the desired range. However, the largest diaphragm $(3 \mathrm{~cm}$ sides) had a very low resonance frequency while the smaller diaphragm $(1 \mathrm{~cm}$ sides $)$ had smaller deflection. In order to balance between the output signal and the resonance frequency, we selected the $2 \mathrm{~cm}$ size for the sensor. Thus, we made a sensor with $2 \mathrm{~cm}$ sides in a square shape. To test the sound response of the $2 \mathrm{~cm}$ sized sensor, we played sounds of varying frequencies $(100-1000 \mathrm{~Hz})$ at a distance of 2 $\mathrm{mm}$ in front of the diaphragm. The $2 \mathrm{~mm}$ distance was chosen to mimic the gap that should be maintained between human skin (a conductor) and the sensor in order to keep the capacitance of 


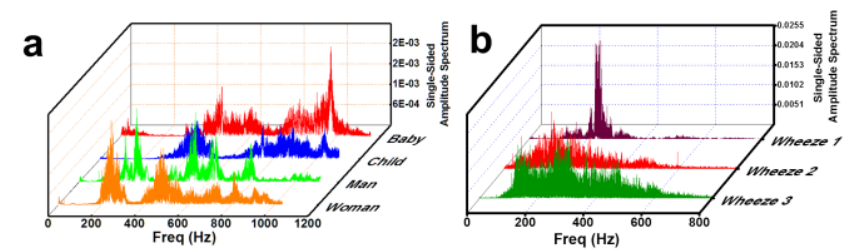

C

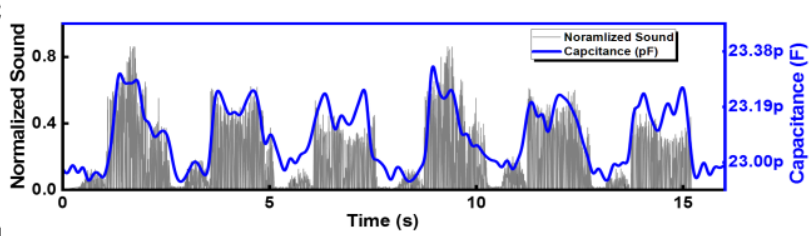

d
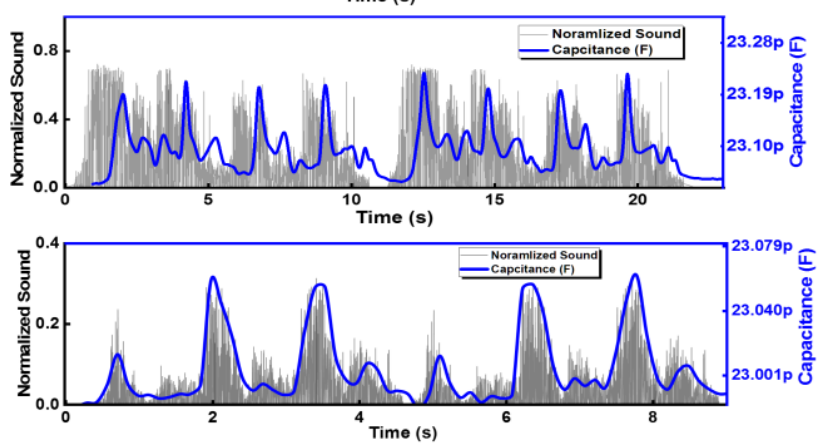

Fig. 3. (a) FFT of sound samples acquired from a baby, child, man, and woman to find the frequency distribution of sound samples for different ages and genders. (b) FFT of three wheezing sound samples to observe the dominant frequency pattern. (c-e) Three wheezing sound samples (blue data) acquired from the sensor compared with the respective original sounds (grey data). The data in blue represents the signal from our sensor, while the grey data show the signal plots of the original sound files. [1]

the diaphragm from changing. The frequency at which we reached maximum amplitude was taken as the resonance frequency. Fig. 2c shows the results from the frequency sweep where the strong response is observed in the range of 200-500 $\mathrm{Hz}$. The amplitude of output escalates after $200 \mathrm{~Hz}$, peaking at $250 \mathrm{~Hz}$. The output remains high until $450 \mathrm{~Hz}$. Fig. 2d shows the response of the sensor to different sound frequencies. We see large output signals at 250,300, and $350 \mathrm{~Hz}$. The output at 250 $\mathrm{Hz}$ shows the acoustic resonance pattern. Studies have shown that the median frequencies of wheezing lie within the range of $200-400 \mathrm{~Hz}[39,40]$. Thus, our design is optimal to detect wheezing and uses recyclable low-cost materials. Fig. 2(e) shows the response of the sensor for varying sound intensity ( $300 \mathrm{~Hz}$ sinusoidal tone) played in front of the sensor. Another advantage of the sensor design becomes eminent as it does not respond to sounds below $55 \mathrm{~dB}$. Background noise in a noisy environment can go as high as $50 \mathrm{~dB}$. The sensitivity calculated from the 55-90 dB linear output response region comes out as $64.3 \mathrm{fF} / \mathrm{dB}$. Given the $10 \mathrm{fF}$ resolution of the PSOC BLE, we can detect changes in sound to as low as $0.156 \mathrm{~dB}$.

\section{RESULTS AND DISCUSSION}

\section{A. Analyzing Sounds}

The sensor is attached to the chest in order to facilitate early diagnosis preemptive detection of an asthma attack. Although the sensor is designed to respond to a limited frequency range of $100-600 \mathrm{~Hz}$, other chest sounds like talking, coughing, and breathing has low-frequency components that interfere with the wheezing signals. Thus, a method needs to be developed to separate wheezing sounds from the various forms of noise originating from the human chest. To investigate
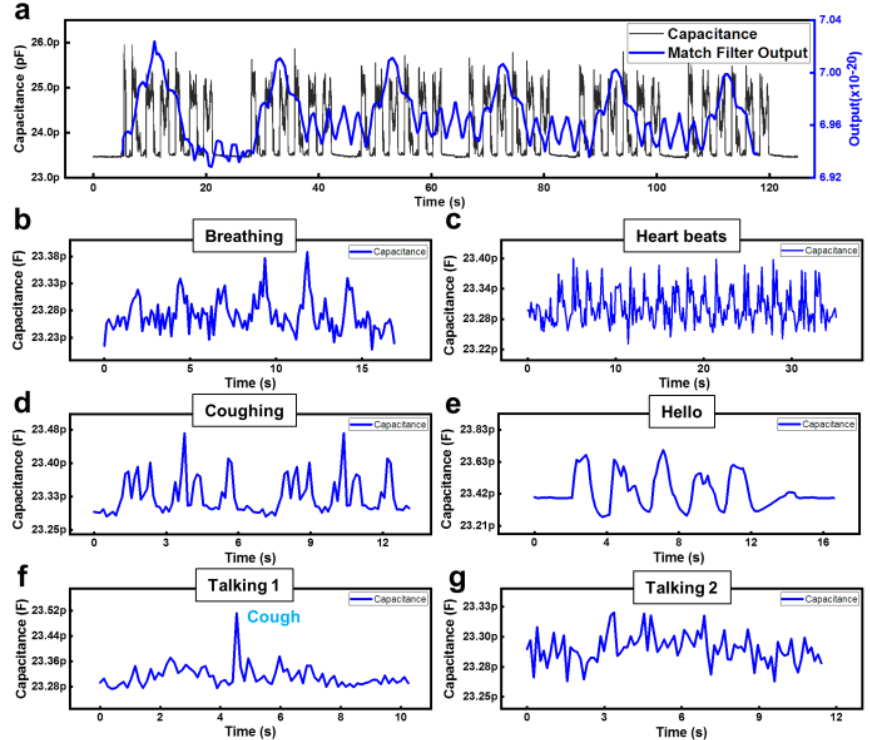

Fig. 4. (a) Data recorded when wheezing sound 1 is replayed. Samples of (b) breathing, (c) heartbeats, (d) coughing, (e) saying hello, and (f, g) talking sounds acquired from a 28 year old adult male. [1]

the effect of talking, we observed the frequency spectrum of different sound samples taken from four subjects: a 1-year-old girl, a 10-year-old boy, an adult man, and an adult woman. The sound samples were recorded by placing a smartphone microphone on the chest of the subjects. The frequency spectrum is created in MATLAB using a Fast Fourier Transformation (FFT), a popular method used to extract features from sounds (Fig. 3a) [41]. Similarly, we observed the FFT of 3 different chest-wheezing sounds taken from the archives of KAUST Medical Clinic (Fig. 3b). It is evident that the most frequency components of wheezing lie below $600 \mathrm{~Hz}$. In turn, younger subjects have frequency components predominantly above $600 \mathrm{~Hz}$. As our sensor is designed to resonate between $250 \mathrm{~Hz}$ to $400 \mathrm{~Hz}$, we form a mechanical high pass filter as the sensor would largely respond to wheezing (lower frequency) sounds while ignoring sounds from the vocal cords, especially for a child (higher frequency).

\section{B. Response to Wheezing and Everyday Sounds}

We setup an experiment to test the performance of our design as an acoustic sensor. A speaker was used to play wheezing sounds with an intensity of $80 \mathrm{~dB}$ at a $2 \mathrm{~mm}$ distance from the sensor. The results were then plotted and compared with the output profile of the actual wheezing sounds (Figs. 3ce). The sensor can successfully extract sufficient features from the wheezing sounds such that they are differentiable from each other. In order to check the repeatability of the sensor detection, we played the wheezing 1 sound repeatedly in front of the sensor; the results can be seen in Fig. 4a. The peaks of the matched filter output for the repeated signal shows that the sensor is able to record sounds with the consistent accuracy. The Gage R\&R (gage repeatability and reproducibility) statistical tool calculates the Repeatability (\%Var) as $92.12 \%$.

\section{Wheeze Detection Using Matched Filter}

Wheezing can be detected by looking for peaks in the frequency domain and they are generally seen as fine peaks in the power spectrum under $2000 \mathrm{~Hz}$ in the spectral analysis [42]. The obstructed and irregular airflow of wheezing improves the better signal-to-noise ratio (SNR) compared to a normal airflow 

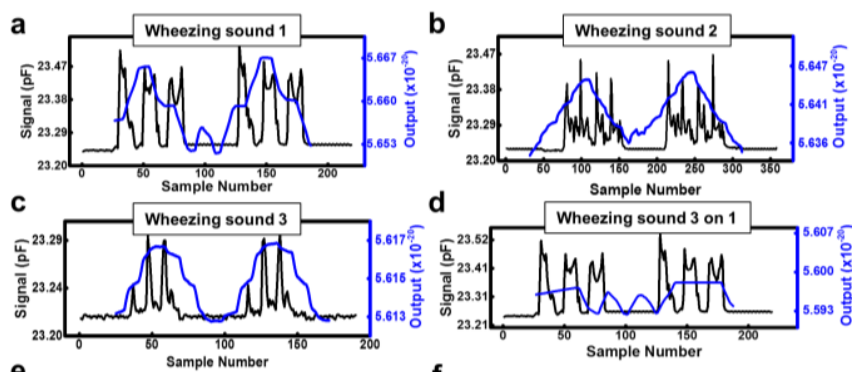

Wheezing sound 3 on 1
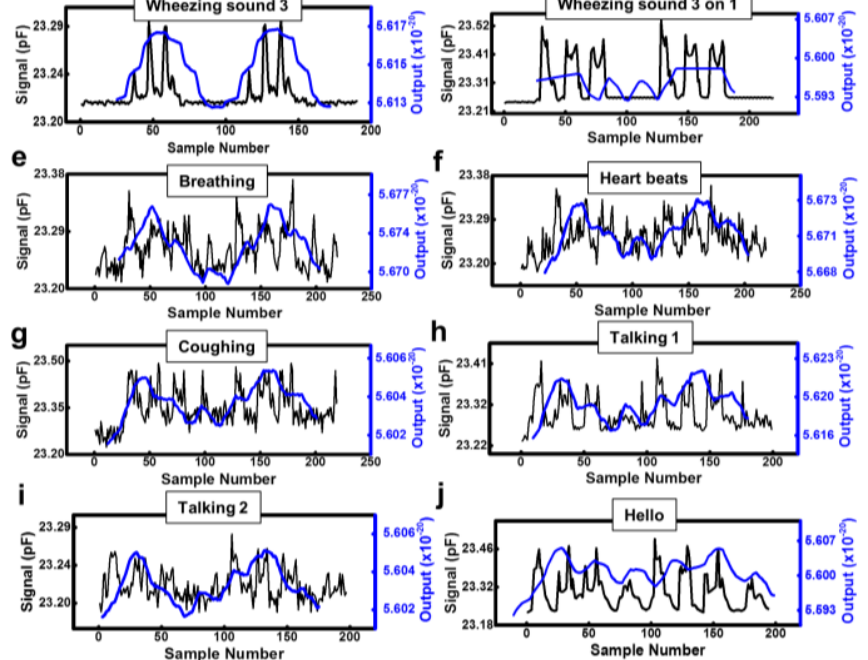

Sample Number

Hello

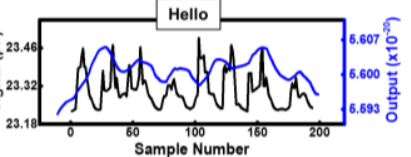

Fig. 5. (The data in blue is the output plot of matched filter while the data on black is the sound signals acquired from our sensors superimposed with various noise sounds identified by the label on each graph.) Matched filter output for wheezing sounds (a) 1 , (b) 2 , and (c) 3 with the peaks representing a positive detection of a signal similar to the template. (d) Matched filter output for wheezing sound 3 on wheezing sound 1 showing no similar peaks, reflecting the dissimilar signals. (e) Breathing, (f) heart beating, (g) coughing, and (h, i) talking sounds superimposed on wheezing sound 1 , and subsequent matched filter output with peaks showing a positive detection of wheezing in the noisy signal. (j) Matched filter output for repeated hello sounds. (Modified form [1])

breath sound [43]. However, sounds coming from the human chest, including breathing, cough, heartbeats, crackles, and talking still interfere with wheezing detection [39, 41]. Especially, talking constitutes lower frequency sounds originating from the vocal cord, which traverses through the chest muscles and reaches the sensor. We used our sensor attached to a human chest to gather sound plots of; breathing, heartbeats, coughing, repeated hello sounds, male talking with a cough in-between, and female talking (Figs. 4b-g). We superimposed all of these sounds on the wheezing 1 sound to mimic a noisy real-life scenario. The ability to detect wheezing from a noisy signal is a crucial criterion to form a robust wheeze-detection system. Matched filters are generally used to identify a known signal or template in an unknown signal. The matched filter can improve noise rejection and extract features out of a noisy signal Matched filters have been extensively used for the detection of blood vessels, ECG peaks, seismic events, moving targets, cracks, SONAR, and microfluidic cells [44-51]. It is similar to the convolution of an unknown signal with a conjugated time-reversed version of the template. It is the optimal linear filter for maximizing the signal-to-noise ratio (SNR) in the presence of additive stochastic noise. This method commonly used to detect backscattered signals in radar. The backscattered signal is matched with the template of the sent signal and, if the matched filter output shows a peak, the filter can determine that the transmitted signal has returned. The matched filter output produces a visible peak when a signal similar to the template is detected in a noisy signal [52]. We have included a supplementary color MPEG file (S2) which shows the implementation of a matched filter where a signal template is correlated with a noisy signal containing a signal matching the a

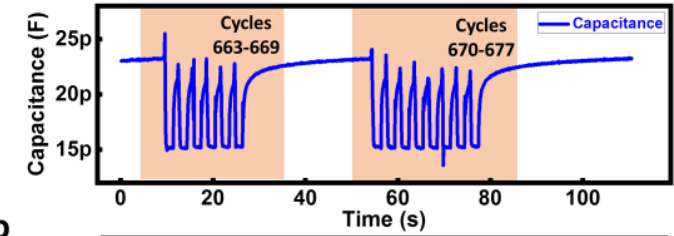

b

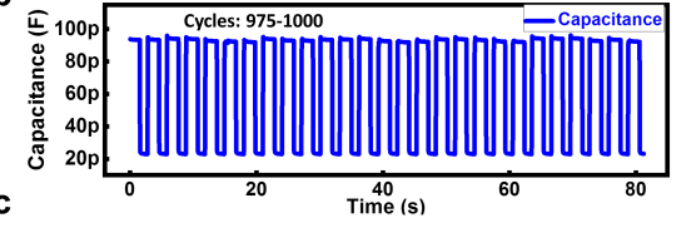

c

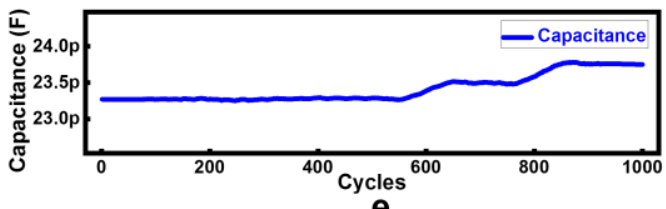

d

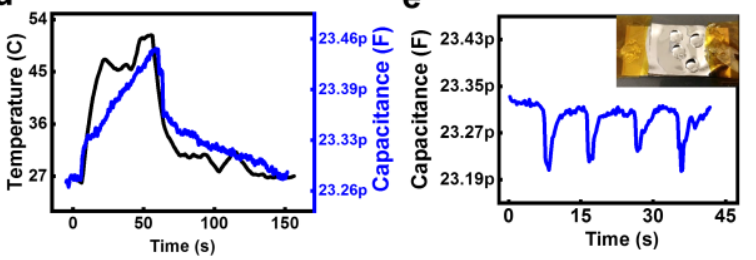

Fig. 6. (a) Output of sensor from cycles 663 to 677 of the $5 \mathrm{~mm}$ bending radius test. (b) Output of sensor from cycles 975 to 1000 of the cyclic pressure test. (c) Changes in the output of sensor from cycles zero to 1000 of the cyclic pressure test. (d) Sensor response to heating and cooling. (e) Sensor response to application of salt water (having equivalent composition of sweat) on the surface of the diaphragm.

template. This is available at http://bit.ly/2Ks4Weo. The template was embedded in the middle of the noisy signal and, when the template passed the point where the signal was embedded in noise, a peak in the output flags the positive detection of a signal similar to the template. The previously acquired signals from Fig. 3a are used as templates for the implementation of the matched filter. We further acquired signals of three different kinds of wheezing sound where each wheezing sound was played twice in succession. These templates of each wheezing sound were used to perform matched filtering and the results can be seen in Figs. 5a-c. It can be seen that the output in blue clearly shows a peak indicating a positive detection. When the wheezing 3 template was used to perform match filtering on the wheeze 1 signal, the output did not show differentiable peaks, as shown in Fig. 5d. This confirms that one wheezing template only gives positive detection for the same type of wheezing which helps to differentiate between different types of wheezing across various human subjects provided that a template for each type of wheezing is available. Subsequently, the template of wheeze 1 sound was used to perform matched filtering on the noisy signals where wheeze 1 sounds are buried in various chest sounds (Figs. 4a-f). The results are plotted in Figs. 5e-i. Visible peaks show that matched filter was able to successfully detect wheezing from the noisy signal even though the wheeze 1 signal is not visible to naked eye when buried in the noisy signal. One failure scenario was observed when the repeated hello sounds from Fig. 4e was superimposed on the wheeze 1 sounds; the match filter was unable to create differentiable peaks (Fig. 5j). The repeated hello sounds were similar in shape to the wheeze 1 sound, thus resulting in an incomprehensible detection. Other sounds that have a comparable output waveform to a wheezing signal may result in a false detection. 

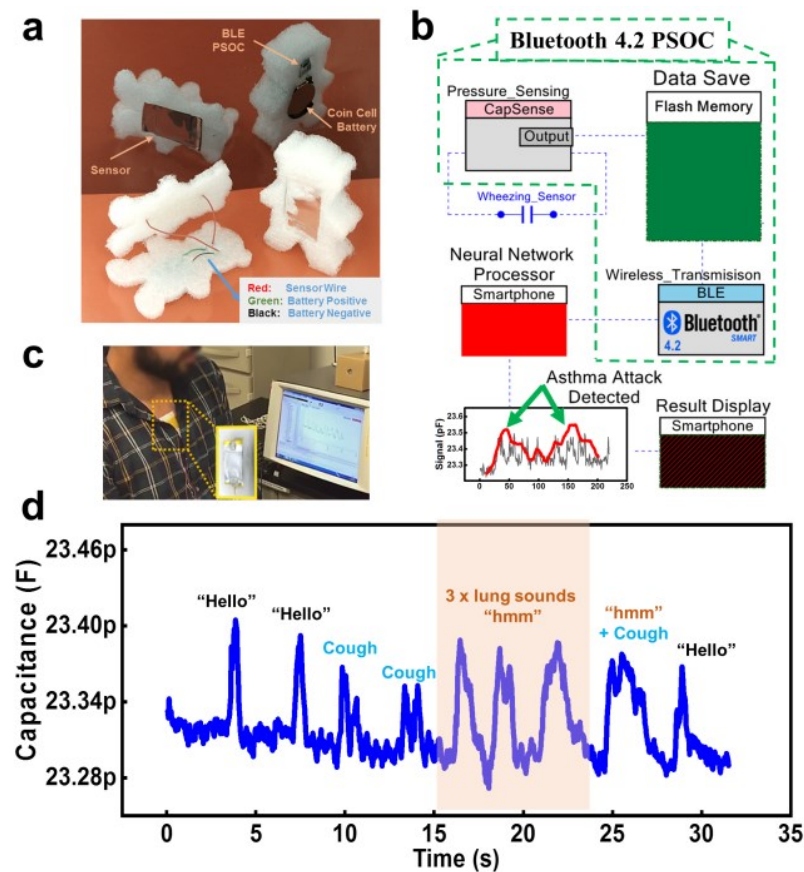

Fig. 7. (a) Sensor shown in Styrofoam package (left) and the wireless PSoC system on the backside (right). (b) Schematic of the wireless PSOC showing the internal features being used and data delivery to a peripheral, like a smartphone or PC, for further signal processing and asthma detection in real time. (c) Real time data acquisition from the chest of a human subject. (c) A sensor attached like a stethoscope to chest with real-time data result plotting. (d) The real-time output of sensor obtained from the chest of the human subject asked to make humming sounds, do coughing, and talking.

\section{Bending, Pressure, Temperature, and Humidity Testing}

Since the sensor needs to be worn on the chest, it must be able to withstand external forces other than sounds, like bending, human handling, varying temperatures, and sweat. In order to test the sensor's performance when bent, we subjected the sensor to 700 cycles of bending the radius of $5 \mathrm{~mm}$. Fig. 6a shows the output of the sensor from cycles 663-669 and 670677 . It can be seen that upon releasing the structure between cycles 669-670, the sensor fully recovers to its initial capacitance. At the end of 700 cycles, the sensor fully returned to its original condition. This shows that the strong material of the diaphragm materials retained their properties even after being subjected to extreme bending conditions.

Furthermore, we put the sensor through 1000 cycles of highpressure cyclic testing. The applied force was analogous to a finger touch. The force applied by sound lies in the $<1 \mathrm{~Pa}$ range, but rough human handling can reach as high as a few $\mathrm{MPa}$ [53], which means the sensor is comparatively much less likely to be affected by sound pressure than human handling. The change in capacitance for loud sounds was just a few hundred femtofarads, but the output rises to as much as $100 \mathrm{pF}$ when we subjected the sensor to a repeated force of $1 \mathrm{MPa}$. In order to test for the strength of the sensor in harsh conditions such as pressing with a finger, we subjected the sensor to a repeated force of $1 \mathrm{MPa}$, which is equivalent to a finger poke of $60 \mathrm{~N}$ force on a $0.5 \mathrm{~cm}^{2}$ surface area. Fig. 6b shows the output from cycles 975-1000. Fig. 6c shows how the sensor's output varied over the course of 1000 cycles of extreme pressure application. The results confirmed that the sensor maintained its mechanical integrity after hundreds of cycles. The sensor underwent a total change of $0.51 \mathrm{pF}$ at the end of 1000 cycles with a standard deviation of $0.19 \mathrm{pF}$. We have included a supplementary color MPEG file
(S3) that shows the bending and pressure testing of the sensors in real time using a wirelessly controlled $3 \mathrm{D}$ printed bending platform. This is available at http://bit.ly/2Ks4Weo. Next, we subject the sensor to a temperature test. The sensor was heated from room temperature to $47^{\circ} \mathrm{C}$; the results are plotted as shown in Fig. 6d. The capacitance of the sensor increased with temperature due to an increase in the resistance of the $\mathrm{Al}$ component $[54,55]$. However, the capacitance returned to its original value as the sensor cooled to room temperature. To test resistance to sweat exposure, we first prepare a sample of water with a similar salt concentration of sweat. The results are plotted in Fig. 6e. The blowout images show the drops of salt water on the sensor. The salt water drops had no effect on the output of the sensor. The momentary drop in capacitance was due to the proximity of the hand holding the pipette full of salt water [5658]. The capacitance returns its original value after the hand is moved away from the sensor.

After each of these tests, the sensor recovered its initial capacitance. Even when the absolute value of the capacitance changes under the various conditions, e.g., bending, high temperature, and sweat exposure, the ability to sense sounds remained unaffected as the sounds will create a relative change in capacitance on top of the existing absolute value. The effect of absolute change in capacitance can be accounted for by using baseline correction algorithms, such as those used with sensors that are affected by environmental conditions [59-63], to adjust the baseline value at regular time intervals.

\section{E. Placement on Human Subject and Test Trial}

Different studies have used a combination of ECG and photoplethysmogram (PPG) to detect wheezing [11], but due to the nature of ECG and PPG, they require the use of gels, adhesives patches, and rigid sensors to take data from the subject, causing discomfort and requiring regular replacement. We wanted to make a device using our sensor that was small, non-intrusive, and easy to attach to the subject. Styrofoam was used as the packaging material for both comfort and its ability to attenuate unnecessary sounds from the environment (Fig. 7a). [64-66]. The flexibility of our sensor allows it to mold according to the shape of the muscle in a completely non-intrusive way. This device is essentially acts as a standalone add-on. Such addon devices have shown to enhance the functionality of plants, marine animals, and everyday objects [67-70]. Further information about the electronic interface is available in Supplementary Text which can be found at http://bit.ly/2Ks4Weo. In order to demonstrate the functionality of our sensor in real time, we attached the packaged sensor to the chest of a 28-year-old male subject. The subject was asked to make different sounds from lungs like cough and imitate wheezing sounds by making humming sounds. We have included a supplementary color MPEG file (S4) showing the human subject test trial experiment. This is available at http://bit.ly/2Ks4Weo. The subject can be seen making lung sounds and saying "hello" while the result is displayed in real time on the monitor (Fig. 7c). The output plot from the same test can be seen in Fig. 7d. It shows that the sensor can detect different occurrences of a cough, hello sound, and humming sound from the lungs (similar to wheezing sounds). Studies have been conducted to find the best possible place for respiratory disease detection and optimal sound acquisition $[7,11,12,71]$. The chest is found to be the most useful place for respiratory disease detection. For the process to be run in real time when the sensor is to be placed on a human chest, an experimental setup 
was established to transfer the data directly from the sensor's primary Bluetooth module to a secondary Bluetooth module. This secondary Bluetooth module transfers the data to MATLAB running on a PC over UART where the data is processed in real time for wheezing detection. A schematic of the electronic system scheme that can be used for detection of asthma in real time can be seen in Fig. $7 \mathbf{b}$.

\section{CONCLUSION}

We have proven the utility and versatility of our low-cost sensor for the potential application of wheezing episode detection in point-of-care diagnostics. We demonstrate a proofof-concept device with a specialized design to detect wheezing while being attached to the chest of a human. The cost and complexity of the manufacturing process are low because of the use of sustainable materials and a scalable manufacturing process. The circuit is less complicated compared to the alternatives, resulting in a reduced size and lower power consumption of the overall system. The smart design combined with the matched filter implementation forms a robust wheezing detection system that can detect wheezing among several reallife noise case scenarios. The flexibility of the sensor allows it to conform to irregular shapes so that it can be attached nonintrusively to the human chest. The low-cost sensor allows for integration at a large scale in the healthcare industry for realtime monitoring of patients that are at a risk for becoming asthmatic. The sensors are interfaced with Bluetooth enabled chip, which further allows integration in the Internet of Things (IoT) enabled systems for early intervention when a patient is having an asthma attack.

\section{References}

[1] M. M. H. S. M. Khan, "Low-cost Foil based Wearable Sensory System for Respiratory Sound Analysis to Monitor Wheezing," in 2019 IEEE 16th International Conference on Wearable and Implantable Body Sensor Networks (BSN), 2019, pp. 1-4: IEEE. doi: 10.1109/BSN.2019.8771076

[2] C. Lai, R. Beasley, J. Crane, S. Foliaki, J. Shah, and S. Weiland, "Global variation in the prevalence and severity of asthma symptoms: phase three of the International Study of Asthma and Allergies in Childhood (ISAAC)," Thorax, 2009. doi: 10.1136/thx.2008.106609

[3] J. A. Castro-Rodríguez, C. J. Holberg, A. L. Wright, and F. D. Martinez, "A clinical index to define risk of asthma in young children with recurrent wheezing," American journal of respiratory and critical care medicine, vol.162, no. 4, pp. 1403-1406, 2000. doi: 10.1164/ajrccm.162.4.9912111

[4] K. D. van de Kant, E. M. Klaassen, Q. Jöbsis, A. J. Nijhuis, O. C. van Schayck, and E. Dompeling, "Early diagnosis of asthma in young children by using non-invasive biomarkers of airway inflammation and early lung function measurements: study protocol of a case-control study," BMC public health, vol. 9 , no. 1, p. 210, 2009. doi: 10.1186/1471-2458-9-210

[5] B.-S. Lin, H.-D. Wu, and S.-J. Chen, "Automatic wheezing detection based on signal processing of spectrogram and back-propagation neural network," Journal of healthcare engineering, vol. 6, no. 4, pp. 649-672, 2015. doi: 10.1260/2040-2295.6.4.649

[6] J. Zheng, C. Ha, and Z. Zhang, "Design and evaluation of a ubiquitous chest-worn cardiopulmonary monitoring system for healthcare application: a pilot study," Medical \& biological engineering \& computing, vol. 55, no. 2, pp. 283-294, 2017. doi: 10.1007/s11517-0161518-5

[7] S. M. Shaharum, K. Sundaraj, and R. Palaniappan, "Tracheal sound reliability for wheeze data collection method: A review," in Control System, Computing and Engineering (ICCSCE), 2012 IEEE International Conference on, 2012, pp. 264-267: IEEE. doi: 10.1109/ICCSCE.2012.6487153

[8] N. Meslier, G. Charbonneau, and J. Racineux, "Wheezes," European respiratory journal, vol. 8, no. 11, pp. 1942-1948, 1995. doi:

[9] S. A. Taplidou and L. J. Hadjileontiadis, "Wheeze detection based on time-frequency analysis of breath sounds," Computers in biology and medicine, vol. 37, no. 8, pp. 1073-1083, 2007. doi: 10.1016/j.compbiomed.2006.09.007

[10] J. Dieffenderfer et al., "Low-Power Wearable Systems for Continuous Monitoring of Environment and Health for Chronic Respiratory Disease," IEEE journal of biomedical and health informatics, vol. 20, no. 5, pp. 1251-1264, 2016. doi: 10.1109/JBHI.2016.2573286

[11] Y. Zhong, Y. Pan, L. Zhang, and K.-T. Cheng, "A wearable signal acquisition system for physiological signs including throat PPG," in Engineering in Medicine and Biology Society (EMBC), 2016 IEEE 38th Annual International Conference of the, 2016, pp. 603-606: IEEE. doi: 10.1109/EMBC.2016.7590774

[12] Y. Wang et al., "Ultra-sensitive graphene strain sensor for sound signal acquisition and recognition," Nano Research, vol. 8, no. 5, pp. 1627-1636, 2015. doi: 10.1007/s12274-014-0652-3

[13] Y. Tai, M. Mulle, I. A. Ventura, and G. Lubineau, "A highly sensitive, low-cost, wearable pressure sensor based on conductive hydrogel spheres," Nanoscale, vol. 7, no. 35, pp. 14766-14773, 2015. doi: $10.1039 /$ C5NR03155A

[14] H. Pasterkamp, S. S. Kraman, and G. R. Wodicka, "Respiratory sounds: advances beyond the stethoscope," American journal of respiratory and critical care medicine, vol. 156, no. 3, pp. 974-987, 1997. doi: 10.1164/ajrccm.156.3.9701115

[15] M. Folke, L. Cernerud, M. Ekström, and B. Hök, "Critical review of noninvasive respiratory monitoring in medical care," Medical and Biological Engineering and Computing, vol. 41, no. 4, pp. 377-383, 2003. doi: 10.1007/BF02348078

[16] I. Sen and Y. Kahya, "A multi-channel device for respiratory sound data acquisition and transient detection," in Engineering in Medicine and Biology Society, 2005. IEEE-EMBS 2005. 27th Annual International Conference of the, 2006, pp. 6658-6661: IEEE. doi: 10.1109/IEMBS.2005.1616029

[17] X. Huo and M. Ghovanloo, "A wireless pharmaceutical compliance monitoring system based on magneto-inductive sensors," IEEE Sensors Journal, vol. 7, no. 12, pp. 1711-1719, 2007. doi: 10.1109/JSEN.2007.909233

[18] Y. M. Choi, T. Olubanjo, A. Farajidavar, and M. Ghovanloo, "Potential barriers in adoption of a medication compliance neckwear by elderly population," in 2013 35th Annual International Conference of the IEEE Engineering in Medicine and Biology Society (EMBC), 2013, pp. 46784681: IEEE. doi: 10.1109/EMBC.2013.6610591

[19] P. R. Scheeper et al., "A new measurement microphone based on MEMS technology," Journal of Microelectromechanical systems, vol. 12, no. 6, pp. 880-891, 2003. doi: 10.1109/JMEMS.2003.820260

[20] S. C. Ko, Y. C. Kim, S. S. Lee, S. H. Choi, and S. R. Kim, "Micromachined piezoelectric membrane acoustic device," Sensors and Actuators A: Physical, vol. 103, no. 1-2, pp. 130-134, 2003. doi: 10.1016/S0924-4247(02)00310-2

[21] H. Wang, X. Wang, C. He, and C. Xue, "Design and performance analysis of capacitive micromachined ultrasonic transducer linear array," Micromachines, vol. 5, no. 3, pp. 420-431, 2014. doi 10.3390/mi5030420

[22] K. Marten and P. Marler, "Sound transmission and its significance for animal vocalization," Behavioral ecology and sociobiology, vol. 2, no. 3, pp. 271-290, 1977. doi: 10.1007/BF00299740

[23] H. Nishiyama and M. Nakamura, "Capacitance of a strip capacitor," IEEE Transactions on Components, Hybrids, and Manufacturing Technology, vol. 13, no. 2, pp. 417-423, 1990. doi: 10.1109/33.56178

[24] T. Tsuchiya and H. Funabashi, "A z-axis differential capacitive SOI accelerometer with vertical comb electrodes," Sensors and Actuators A: Physical, vol. 116, no. 3, pp. 378-383, 2004. doi: 10.1016/j.sna.2004.05.008

[25] W. Ye, S. Mukherjee, and N. C. MacDonald, "Optimal shape design of an electrostatic comb drive in microelectromechanical systems," Journal of microelectromechanical systems, vol. 7, no. 1, pp. 16-26, 1998. doi: $10.1109 / 84.661380$

[26] D. Oletic and V. Bilas, "Energy-efficient respiratory sounds sensing for personal mobile asthma monitoring," Ieee sensors journal, vol. 16, no. 23, pp. 8295-8303, 2016. doi: 10.1109/JSEN.2016.2585039

[27] T. WILLIS, "The Importance of Thickness Factors and Flexibility of Design," Clinical and Experimental Optometry, vol. 45, no. 7, pp. 206209, 1962. doi: 10.1111/j.1444-0938.1962.tb01671.x

[28] Y.-S. Kang and P. S. Ho, "Thickness dependent mechanical behavior of submicron aluminum films," Journal of Electronic Materials, vol. 26, no. 7, pp. 805-813, 1997. doi: 10.1007/s11664-997-0255-9

[29] G. Mearini and R. Hoffman, "Tensile properties of aluminum/alumina multi-layered thin films," Journal of Electronic Materials, vol. 22, no. 6, pp. 623-629, 1993. doi: 10.1007/BF02666408 
[30] R. Khakpour, S. R. Mansouri, and A. Bahadorimehr, "Analytical comparison for square, rectangular and circular diaphragms in MEMS applications," in Electronic Devices, Systems and Applications (ICEDSA), 2010 Intl Conf on, 2010, pp. 297-299: IEEE. doi: 10.1109/ICEDSA.2010.5503057

[31] A. Nallathambi and T. Shanmuganantham, "Design of Diaphragm Based MEMS Pressure Sensor with Sensitivity Analysis for Environmental Applications," Sensors \& Transducers, vol. 188, no. 5, p. 48, 2015. doi:

[32] N. R. Council, Low-frequency sound and marine mammals: Current knowledge and research needs. National Academies Press, 1994.

[33] T. Mellow and L. Kärkkäinen, "On the sound field of a circular membrane in free space and an infinite baffle," The Journal of the Acoustical Society of America, vol. 120, no. 5, pp. 2460-2477, 2006. doi: 10.1121/1.2354041

[34]https://www.prosoundweb.com/channels/recording/size_matters_the_diff erences in large- and small-diaphragm microphones/\#

[35] X. Wang, B. Li, O. L. Russo, H. T. Roman, K. K. Chin, and K. R. Farmer, "Diaphragm design guidelines and an optical pressure sensor based on MEMS technique," Microelectronics journal, vol. 37, no. 1, pp. 50-56, 2006. doi: 10.1016/j.mejo.2005.06.015

[36] L. E. Kinsler, A. R. Frey, A. B. Coppens, and J. V. Sanders, "Fundamentals of acoustics," Fundamentals of Acoustics, 4th Edition, by Lawrence E. Kinsler, Austin R. Frey, Alan B. Coppens, James V. Sanders, pp. 560. ISBN 0-471-84789-5. Wiley-VCH, December 1999., p. 560, 1999. doi: N/A

[37] C. Van Mullem, K. Gabriel, and H. Fujita, "Large-Deflection Performance of Surface Micromachined Corrugated Diaphragms," in Proceedings of the 6th International Conference on Solid-State Sensors and Actuators, 1991, pp. 1014-1017.

[38] S. K. Clark and K. D. Wise, "Pressure sensitivity in anisotropically etched thin-diaphragm pressure sensors," IEEE Transactions on Electron Devices, vol. 26, no. 12, pp. 1887-1896, 1979. doi: 10.1109/TED.1979.19792

[39] N. S. Haider and J. Joseph, "An investigation on the statistical significance of spectral signatures of lung sounds," Biomedical Research, 2017.

[40] S.-H. Li, B.-S. Lin, C.-H. Tsai, C.-T. Yang, and B.-S. Lin, "Design of wearable breathing sound monitoring system for real-time wheeze detection," Sensors, vol. 17, no. 1, p. 171, 2017. doi: 10.3390/s17010171

[41] M. Bahoura and C. Pelletier, "New parameters for respiratory sound classification," in Electrical and Computer Engineering, 2003. IEEE CCECE 2003. Canadian Conference on, 2003, vol. 3, pp. 1457-1460: IEEE. doi: 10.1109/CCECE.2003.1226178

[42] J.-C. Chien, H.-D. Wu, F.-C. Chong, and C.-I. Li, "Wheeze detection using cepstral analysis in gaussian mixture models," in Engineering in Medicine and Biology Society, 2007. EMBS 2007. 29th Annual International Conference of the IEEE, 2007, pp. 3168-3171: IEEE. doi: 10.1109/IEMBS.2007.4353002

[43] B.-Y. Lu, L.-C. Huang, L.-M. Hsu, S.-H. Tang, H.-D. Wu, and J.-S. Lai, "Statistical perspective on noise cancellations of wheeze recordings by adjusting the sampling rates of sound card," in International Conference on Information Technology and Applications, Sydney, 2013, pp. 192-195.

[44] S. Chaudhuri, S. Chatterjee, N. Katz, M. Nelson, and M. Goldbaum, "Detection of blood vessels in retinal images using two-dimensional matched filters," IEEE Transactions on medical imaging, vol. 8, no. 3, pp. 263-269, 1989

[45] T. Chanwimalueang, W. von Rosenberg, and D. P. Mandic, "Enabling Rpeak detection in wearable ECG: Combining matched filtering and Hilbert transform," in Digital Signal Processing (DSP), 2015 IEEE International Conference on, 2015, pp. 134-138: IEEE. doi: 10.1109/ICDSP.2015.7251845

[46] R. Buland and F. Gilbert, "Matched filtering for the seismic moment tensor," Geophysical research letters, vol. 3, no. 3, pp. 205-206, 1976. doi: 10.1029/GL003i003p00205

[47] P. M. Shearer, "Global seismic event detection using a matched filter on long - period seismograms," Journal of Geophysical Research: Solid Earth, vol. 99, no. B7, pp. 13713-13725, 1994. doi: 10.1029/94JB00498

[48] S.-W. Xu, P.-L. Shui, X.-Y. Yan, and Y.-H. Cao, "Combined adaptive normalized matched filter detection of moving target in sea clutter," Circuits, Systems, and Signal Processing, vol. 36, no. 6, pp. 2360-2383, 2017. doi: 10.1007/s00034-016-0413-5

[49] L. Gao, B. Yang, Q. Du, and B. Zhang, "Adjusted spectral matched filter for target detection in hyperspectral imagery," Remote Sensing, vol. 7, no. 6, pp. 6611-6634, 2015. doi: 10.3390/rs70606611

[50] Z. Zhao, A. Zhao, J. Hui, B. Hou, R. Sotudeh, and F. Niu, "A FrequencyDomain Adaptive Matched Filter for Active Sonar Detection," sensors, vol. 17 , no. 7 , p. 1565 , 2017. doi: $10.3390 / \mathrm{s} 17071565$

[51] R. Liu et al., "Design and modeling of electrode networks for codedivision multiplexed resistive pulse sensing in microfluidic devices," Lab on a Chip, vol. 17, no. 15, pp. 2650-2666, 2017. doi: 10.1039/C7LC00545H

[52] G. Turin, "An introduction to matched filters," IRE transactions on Information theory, vol. 6, no. 3, pp. 311-329, 1960.

[53] A. D. Astin, "Finger force capability: measurement and prediction using anthropometric and myoelectric measures," Virginia Tech, 1999. doi:

[54] A. Association, Aluminum: properties and physical metallurgy. ASM International, 1984

[55] J. R. Davis, Aluminum and aluminum alloys. ASM international, 1993.

[56] J. M. Nassar, K. Mishra, K. Lau, A. A. Aguirre - Pablo, and M. M. Hussain, "Recyclable Nonfunctionalized Paper - Based Ultralow - Cost Wearable Health Monitoring System," Advanced Materials Technologies, vol. 2, no. 4, 2017. doi: 10.1002/admt.201600228

[57] J. M. Nassar and M. M. Hussain, "Impact of Physical Deformation on Electrical Performance of Paper-Based Sensors," IEEE Transactions on Electron Devices, vol. 64, no. 5, pp. 2022-2029, 2017. doi: 10.1109/TED.2017.2650981

[58] J. M. Nassar et al., "Paper skin multisensory platform for simultaneous environmental monitoring," Advanced Materials Technologies, vol. 1, no. 1, 2016. doi: 10.1002/admt.201600004

[59] T. Li, D. A. Sobel, and S. V. Joshi, "Waterproof baseline tracking in capacitive touch controllers," ed: Google Patents, 2014.

[60] J. Liu and J. L. Koenig, "A new baseline correction algorithm using objective criteria," Applied spectroscopy, vol. 41, no. 3, pp. 447-449, 1987. doi: $10.1366 / 0003702874449110$

[61] N. Reinecke and D. Mewes, "Recent developments and industrial/research applications of capacitance tomography," Measurement Science and Technology, vol. 7, no. 3, p. 233, 1996.

[62] W. C. Westerman, "Periodic sensor panel baseline adjustment," ed: Google Patents, 2011.

[63] D. A. Soss, "Sensor baseline compensation in a force-based touch device," ed: Google Patents, 2008.

[64] J. Allard and N. Atalla, Propagation of sound in porous media: modelling sound absorbing materials 2e. John Wiley \& Sons, 2009.

[65] Y. Piedrahita and F. Fajardo, "Construction of an anechoic chamber for characterization of the sound transmission loss," Revista Brasileira de Ensino de Física, vol. 34, no. 4, pp. 1-9, 2012. doi: 10.1590/S180611172012000400005

[66] L. Kokkinidis, R. M. Zacharko, and H. Anisman, "Amphetamine withdrawal: a behavioral evaluation," Life sciences, vol. 38, no. 17, pp. 1617-1623, 1986. doi: 10.1016/0024-3205(86)90501-1

[67] J. M. Nassar, S. M. Khan, D. R. Villalva, M. M. Nour, A. S. Almuslem, and M. M. Hussain, "Compliant plant wearables for localized microclimate and plant growth monitoring," npj Flexible Electronics, vol. 2, no. 1, p. 24, 2018. doi: 10.1038/s41528-018-0039-8

[68] S. M. Khan, S. F. Shaikh, N. Qaiser, and M. M. Hussain, "Flexible Lightweight CMOS-Enabled Multisensory Platform for Plant Microclimate Monitoring," IEEE Transactions on Electron Devices, vol. 65, no. 11, pp. 5038-5044, 2018. doi: 10.1109/TED.2018.2872401

[69] S. F. Shaikh et al., "Noninvasive Featherlight Wearable Compliant " Marine Skin" : Standalone Multisensory System for Deep - Sea Environmental Monitoring," Small, p. 1804385, 2019. doi: 10.1002/smll.201804385

[70] S. Khan, N. Qaiser, and M. M. Hussain, "An inclinometer using movable electrode in a parallel plate capacitive structure," AIP Advances, vol. 9, no. 4, p. 045118 , 2019. doi: 10.1063/1.5092146

[71] H. Kalantarian, N. Alshurafa, T. Le, and M. Sarrafzadeh, "Monitoring eating habits using a piezoelectric sensor-based necklace," Computers in biology and medicine, vol. 58, pp. 46-55, 2015. Doi: 10.1016/j.compbiomed.2015.01.005 